\title{
Seismic wavefield polarization - Part I: Describing an elliptical polarized motion, a review of motivations and methods
}

\author{
Claire Labonne ${ }^{1,2, a}$, Olivier Sèbe ${ }^{1}$, and Stéphane Gaffet ${ }^{2,3}$ \\ ${ }^{1}$ CEA, DAM, DIF, 91297 Arpajon, France \\ ${ }^{2}$ Univ. Nice Sophia Antipolis, CNRS, IRD, Observatoire de la côte d'Azur, Géoazur UMR 7329, \\ Valbonne, France \\ ${ }^{3}$ LSBB UMS 3538, Rustrel, France
}

\begin{abstract}
The seismic wavefield can be approximated by a sum of elliptical polarized motions in 3D space, including the extreme linear and circular motions. Each elliptical motion need to be described: the characterization of the ellipse flattening, the orientation of the ellipse, circle or line in the 3D space, and the direction of rotation in case of non-purely linear motion. Numerous fields of study share the need of describing an elliptical motion. A review of advantages and drawbacks of each convention from electromagnetism, astrophysics and focal mechanism is done in order to thereafter define a set of parameters to fully characterize the seismic wavefield polarization.
\end{abstract}

\section{Introduction}

The seismic wavefield is a combination of polarized waves in the three-dimensional (3D) space. The polarization is a characteristic of the wave related to the particle motion. The displacement of particles effected by elastic waves shows a particular polarization shape and a preferred direction of polarization depending on the source properties (location and characteristics) and the Earth structure. P-waves, for example, generate linear particle motion in the direction of propagation; the polarization is thus called linear. Rayleigh waves, on the other hand, generate, at the surface of the Earth, retrograde elliptical particle motion. Three-component seismic stations record the trajectory of a particle displacement at the free surface of the Earth, and thus the polarization. The change in polarization can be analyzed and interpreted to improve our understanding of both the source process and the Earth structures illuminated by the recorded waves. The objective of the polarization analysis is the determination of the state of polarization consisting in a type of polarization (linear, purely elliptical or circular) and an orientation of the polarization in 3D space. This polarization analysis requires a system of parameters fit to describe an object and its attributes in space. Numerous fields of study share this need. In the following, in order to be able to build an efficient parameter system in seismology, a reviewed convention from other disciplines (electromagnetism, astrophysics and focal mechanism) is done and a parallel between these disciplines and seismology is drawn.

${ }^{a}$ Corresponding author: claire.labonne@cea.fr 


\section{Why an elliptical model?}

A 3-component record of a monochromatic wave can be expressed as followed:

$$
\left(\begin{array}{c}
x \\
y \\
z
\end{array}\right)(t)=\left(\begin{array}{c}
A_{x} \cos \left(2 \pi f t-\varphi_{x}\right) \\
A_{y} \cos \left(2 \pi f t-\varphi_{y}\right) \\
A_{z} \cos \left(2 \pi f t-\varphi_{z}\right)
\end{array}\right)=\underbrace{\left(\begin{array}{c}
A_{x} \cos \varphi_{x} \\
A_{y} \cos \varphi_{y} \\
A_{z} \cos \varphi_{z}
\end{array}\right)}_{\vec{p}} \cos (2 \pi f t)+\underbrace{\left(\begin{array}{c}
A_{x} \sin \varphi_{x} \\
A_{y} \sin \varphi_{y} \\
A_{z} \sin \varphi_{z}
\end{array}\right)}_{\vec{q}} \sin (2 \pi f t)
$$

where $A_{i}$ and $\varphi_{i}$ represent the amplitude and the phase of the component i.

This expression corresponds to the equation of an ellipse as shown in [1]. Thus, the trajectory of a particle through the propagation of a monochromatic wave describes necessarily a full ellipse as a function of time every one time period $\mathrm{T}=1 / \mathrm{f}$. The vectors $\vec{p}$ and $\vec{q}$ can be seen as the positions on the ellipse at $\mathrm{t}=0$ and at $\mathrm{t}=\mathrm{T} / 4$. The extreme cases of linear and circular polarizations will be described by respectively collinear $\vec{p}$ and $\vec{q}$ (linear case) and $|\vec{p}||=| \vec{q}$ (circular case).

Using Fourier analysis, an arbitrary real signal can be decomposed into a sum of quasimonochromatic signal:

$$
\left(\begin{array}{l}
x \\
y \\
z
\end{array}\right)(t)=2 \int_{0}^{\infty} \int_{-\infty}^{+\infty} \vec{p}(f, \tau) \cos (2 \pi f t)+\vec{q}(f, \tau) \sin (2 \pi f t) d \tau d f
$$

where $\vec{p}(f)=\left(\begin{array}{l}\operatorname{Re}(X(f, \tau)) \\ \operatorname{Re}(Y(f, \tau)) \\ \operatorname{Re}(Z(f, \tau))\end{array}\right), \vec{q}(f)=-\left(\begin{array}{l}\operatorname{Im}(X(f, \tau)) \\ \operatorname{Im}(Y(f, \tau)) \\ \operatorname{Im}(Z(f, \tau))\end{array}\right), \quad X(f, \tau)$ corresponds to a local Fourier transform of $\mathrm{x}(\mathrm{t})$ e.g. the Stockwell transform [2], Re and $\mathrm{Im}$ correspond to real and imaginary part operator.

The seismic wavefield records can be decomposed into a sum of quasi-monochromatic waves, and thus a sum of elliptical motions. To describe each elliptical motion, the characterization of the ellipse flattening, the orientation of the ellipse, circle or line in the $3 \mathrm{D}$ space, and the direction of rotation must be defined.

\section{Polarization and orientation conventions in other disciplines}

\subsection{Electromagnetism}

In physics, the notion of polarized waves is not only defined in seismology. In electromagnetism, wave polarization is defined by the temporal behavior of a transverse electromagnetic wave at a given point in space. The state of polarization is investigated in the plane perpendicular to the wave's direction of propagation. Two parameters are introduced to characterize this elliptical state of polarization (see Fig. 1) [1]:

- the signed ellipticity $\left(\boldsymbol{\rho}_{S}\right)$ or the ellipticity angle $(\boldsymbol{\chi}=\operatorname{atan}(\rho))$

This parameter specifies the shape and the direction of rotation. It is defined as the ratio between the semi-minor axis $(\boldsymbol{b})$ and the semi-major axis $(\boldsymbol{a}): \rho_{S}= \pm \frac{b}{a}$. The signed ellipticity values (respectively ellipticity angle) range from -1 to 1 (resp. from $-\frac{\pi}{4}$ to $\frac{\pi}{4}$ ). A zero value (resp. 0) corresponds to a purely linear motion, and $\pm 1\left(\right.$ resp. $\pm \frac{\pi}{4}$ ) to purely circular motion. The sign of $\rho_{S}$ (resp. $\chi$ ) discriminates the two possible directions in which the ellipse may be described: handed-left or handed-right polarization.

- the inclination $\gamma$

This parameter represents the angle between the major direction and the reference direction. The inclination values range from 0 to $\pi$. 


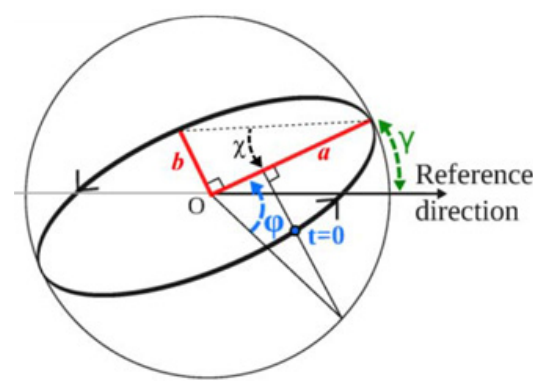

Figure 1. Electromagnetism parameters convention. The blue dot corresponds to the position on the ellipse at time $\mathrm{t}=0$.

Linear and circular polarizations are special cases of elliptical polarization. The "purely elliptical polarization" notion will be used to exclude these two special cases. Each individual state of polarization is defined by a unique pair of shape-orientation parameters $(\chi, \gamma)$. A graphical representation can be done using Poincaré's sphere on which all states of polarization are compressed over the surface of a sphere [3].

In order to reconstruct and not only characterize a polarized motion, one additional parameter needs to be defined, the phase factor $(\varphi)$. The phase permits to locate the point on the ellipse at $\mathrm{t}=0$. Consider a circumscribed circle with a radius of $a$, that is concentric with an ellipse of semi-major axis of $a$. A line perpendicular to the major axis of the ellipse is extended through the point at $\mathrm{t}=0$ and intersects the circle. The phase factor is the angle between the intersect point and the major direction, thus, the eccentric angle and not the polar angle of the position at $\mathrm{t}=0$ relatively to the major direction.

To parametrize an elliptical motion in $2 \mathrm{D}$ plane, four parameters are required: the semimajor axis $a$, the signed ellipticity $\rho_{s}$, the inclination $\gamma$ and the phase factor $\varphi$ :

$$
\left[\begin{array}{l}
x \\
y
\end{array}\right](t)=\left[\begin{array}{cc}
\cos \gamma & -\sin \gamma \\
\sin \gamma & \cos \gamma
\end{array}\right]\left[\begin{array}{c}
a \cos (2 \pi f t-\varphi) \\
\underbrace{a \rho_{s}}_{ \pm b} \sin (2 \pi f t-\varphi)
\end{array}\right]
$$

The parameter system used to describe the polarization in electromagnetism fully characterizes the shape of polarization, the direction of rotation and the orientation of polarization relative to a reference plane. However, this system requires the a-priori knowledge of the orientation of the polarization plane which would be a major limitation in seismology. In seismology, the seismic wavefield is a combination of polarized waves in the 3D space. Due to multi-pathing propagation, a plane of reference cannot be pre-defined. Therefore, polarization parameters need to be oriented in the $3 \mathrm{D}$ space without pre-defined plane of polarization.

\subsection{Astrodynamics}

In a gravitational two-body problem, the relative position of one body with respect to the other follows an elliptical orbit in 3D space [4]. Therefore, the particle motion in seismology can be considered analogous to the elliptical orbit of bodies in space. The relative orbit motion is confined in a plane, but motions in solar system are not confined to a single plane. That is why, in astrodynamics, a 3D representation of an orbit in space is considered (see Fig. 2).

The orbital motion is referred to a reference plane in which the direction of a reference line is defined and called $\mathbf{x}$-direction. The origin of the coordinate system is centered on one 

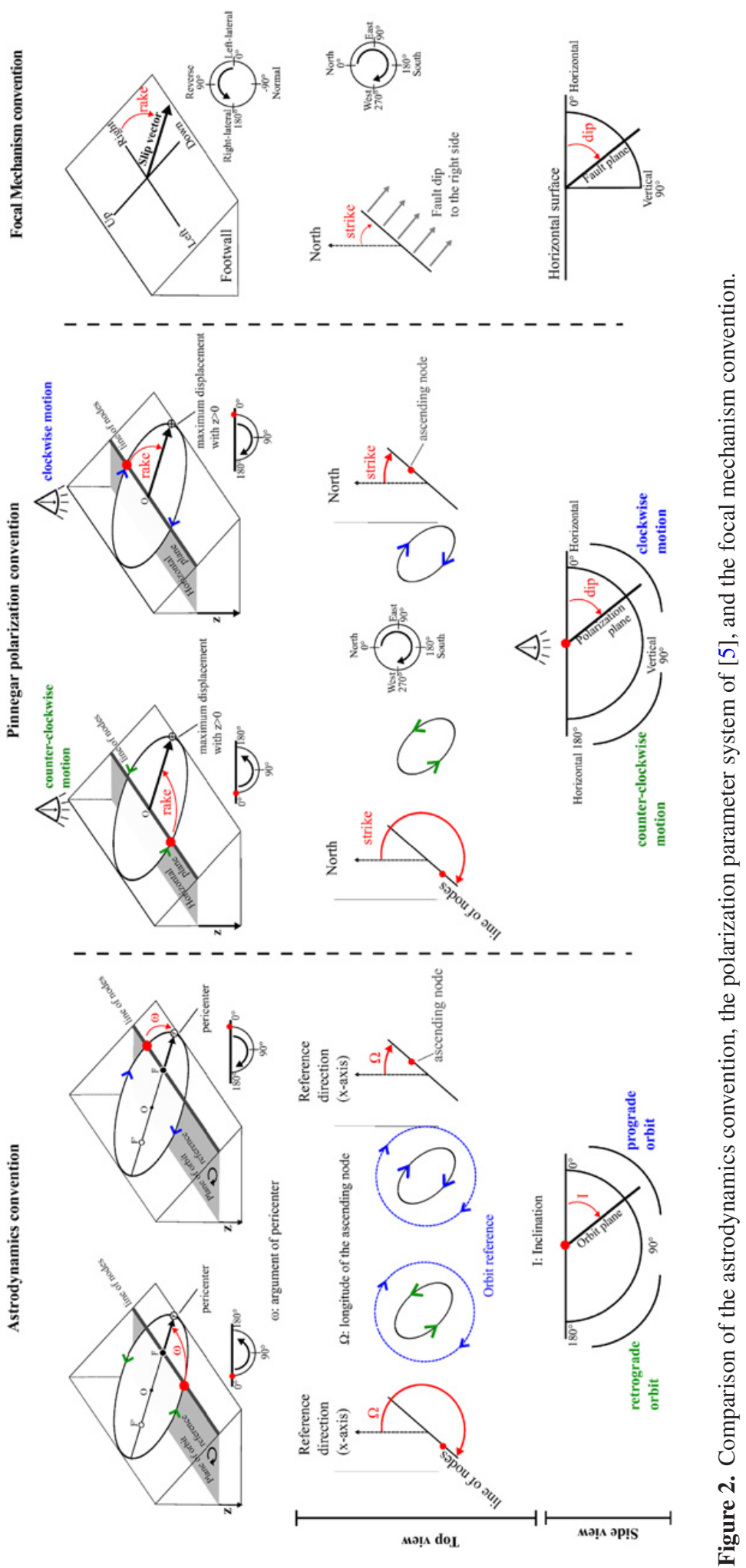
of the two bodies at a focus of the ellipse. Usually, the reference plane is defined as the orbit plane of a reference object. For example, when considering the planets' orbit around the sun, a sun-centered coordinate system is chosen, the reference plane is defined as the Earth's orbit and the reference line is in the direction of the vernal equinox along the line of intersection of the Earth's equator and the Earth's orbit plane. This system can be used to describe any planet's orbit around the sun.

The $\mathbf{z}$-direction is a vector orthogonal to the reference plane, which direction is defined by the rotation of the reference object. In the example mentioned above, the direction of rotation of the Earth is the defining criterion for the positive z-direction.

In general, orbit planes do not coincide with the reference plane but have an inclination angle called inclination of the orbit, I. The line of intersection between an orbital plane and the reference plane is called line of nodes. The point where an orbit crosses the reference plane with positive z-direction is called ascending node. The angle between the reference line and the radius vector of an ascending node is called longitude of the ascending node, $\boldsymbol{\Omega}$. The point corresponding to the minimum orbital radius is called the pericenter. The angle between the radius vector of the ascending node and the pericenter radius is called the longitude of the pericenter, $\omega$.

An orbital plane can be described by a local right-handed triad system (i,j,k). The $\mathrm{i}$-axis is taken to lie along the major axis in the direction of the pericenter. The $\mathrm{j}$-axis is perpendicular to the $\mathrm{i}$-axis and is confined in the orbital plane; its direction is defined by the direction of the rotation. The $\mathrm{k}$-axis is taken perpendicular to the $\mathrm{i}$ - and $\mathrm{j}$-axis.

To summarize, three angles (inclination $I-\operatorname{argument}$ of pericenter $\omega$ - longitude of ascending node $\Omega$ ) are used to relate the local orbital plane system (i,j,k) with a general reference system $(\mathrm{x}, \mathrm{y}, \mathrm{z})$ by a series of three rotations: a rotation around the $\mathrm{z}$ axis with an angle of $\omega$, so that the $\mathrm{x}$-axis coincide with the line of nodes, a rotation around the $\mathrm{x}$-axis with an angle of $I$, so that the two planes are coincident, and finally a rotation around the z-axis with an angle of $\Omega$.

Note that the inclination ranges from $0^{\circ}$ to $180^{\circ}$. If the inclination is smaller than $90^{\circ}$ the motion is prograde whereas if the inclination is larger than $90^{\circ}$ the motion is retrograde. A "purely" elliptical motion in the $3 \mathrm{D}$ space can be fully orientated using this coordinate system.

In seismology, the most complete polarization parametrization uses similar angular convention [5]. The only difference lies in the choice of the origin of the coordinate system defined at the center of the ellipse and not at a focus of the ellipse (Fig. 2). The reference plane is the horizontal plane and the direction of reference is the North (x-axis). The $y$-axis corresponds to the East and the z-vector is oriented downward. Since the origin of the coordinate system is now at the center of the ellipse, a pericenter cannot be defined anymore. In order to be able to orientate the ellipse, the notion of pericenter is replaced by the notion of "positive" maximum displacement which corresponds to the position of maximum displacement in the ellipse with a strictly positive z-coordinate. As in astrodynamics, three angles are defined to relate the local polarization plane system $(\mathrm{i}, \mathrm{j}, \mathrm{k})$ with a general reference system (North, East, Down): the rake, the dip and the strike.

This system still has limitations. A purely horizontal polarization plane is a singularity which cannot be described as both maximum displacements would have the same z-coordinate making them impossible to differentiate. This parameter system requires the definition of both a plane of polarization and a major displacement.

In the case of linear motion, the motion is confined to a line meaning that neither the line of nodes nor the plane of polarization can be defined. Without a line of nodes none of the above mentioned angles exist and a new system would have to be used like the trend and plunge system in structural geology. 
In the case of circular motion, the norm of the displacement vector is constant over the time, thus the position of major displacement cannot be defined and the rake becomes irrelevant. It is worth noting that the rake, the strike and the dip defined above do not exactly correspond to the rake, the strike and the dip used to orientate fault planes in focal mechanism and structural.

\subsection{Focal mechanism}

Fault strike, fault dip, and slip rake are used to describe earthquake focal mechanisms, consisting in fault plane orientation, and the direction of the slip displacement. The fault strike is the azimuth of a strike line, which is the line created by the intersection of a fault plane and a horizontal plane, relative to North. Strike is always defined such that a fault dips to the right side of the trace when moving along the trace in the strike direction. Fault strike values range from $0^{\circ}$ to $360^{\circ}$. The fault dip is the angle between the fault plane and a horizontal plane, with values ranging from $0^{\circ}$ to only $90^{\circ}$. The slip vector shows displacement of the hanging wall relative to the footwall, where the hanging wall is the fault block above the fault plane and the footwall the block below the fault plane. The slip rake is the angle between the strike direction and the slip vector. The rake values ranges from $-180^{\circ}$ to $180^{\circ}$.

Using this system for polarization characterization, the polarization plane corresponds to the fault plane, and the maximum displacement corresponds to the slip displacement. As defined the strike and the dip permit to orientate the plane in space. However, these two angles are not sufficient to characterize the direction of a rotating motion inscribed in the plane and thus orientate the normal vector to this plane. The direction of a rotating motion is an important parameter to characterize the polarization. To give an example, Rayleigh waves usually generate at the surface of the Earth retrograde elliptical particle motion, but the motion recorded at the surface can still sometimes be prograde, as a result of a specific Earth structure below the recorded station.

The major limitation of the focal mechanism system is its inability to characterize a direction of motion contrary to the astrodynamics convention. A comparison of the strike, dip and rake for both conventions is available in Fig. 2.

\section{Conclusion}

The seismic wavefield can be approximated by a sum of elliptical polarized motions in 3D space, including the extreme linear and circular motions. The study of the polarization is a key element in the analysis of source properties and Earth structure. For this study, a system of parameter needs to be defined to characterize the ellipse flattening, the orientation of the ellipse, circle or line in the 3D space, and the direction of rotation in case of nonpurely linear motion. In electromagnetism, the waves' characteristics to be described are very similar to seismic waves'. Therefore, the parameter system would be very well adapted to our needs if it weren't limited by the $2 \mathrm{D}$ space hypothesis. To overcome the $2 \mathrm{D}$ limitation, the astrodynamics provides a full 3D orientation system, presenting the only drawback of having the origin located at a focus of the ellipse instead of the center. [5] proposed a system which regroups the advantage of both electromagnetism and astrodynamics conventions. Finally, [5] system must not be mistaken for the convention used in focal mechanism description. A standardized full parameter system is required in seismology (as defined in [6]) to provide a full characterization of any elliptical motion in 3D space including the extreme linear and circular motions and to provide access to all parameters required in most polarization studies. 


\section{References}

[1] Born, M., Wolf, E. (2000), Principles of optics: electromagnetic theory of propagation, interference and diffraction of light. Cambridge University Press.

[2] Stockwell, R.G., Mansinha, L., \& Lowe, R.P. (1996), Localization of the complex spectrum: the $S$ transform, IEEE transactions on signal processing, 44(3), 998-1001.

[3] Deschamps, G., \& Mast, P. (1973), Poincaré sphere representation of partially polarized fields. IEEE Transactions on Antennas and Propagation, 21(3), 474-478.

[4] Murray, C.D., \& Dermott, S.F. (1999), Solar system dynamics, Cambridge University Press.

[5] Pinnegar, C.R. (2006), Polarization analysis and polarization filtering of threecomponent signals with the time-frequency $\mathrm{S}$ transform. Geophysical Journal International, 165(2), 596-606.

[6] Labonne C., Sèbe O., Gaffet, S., Schindelé, F., Boyer D., Decitre J.B. and Cavaillou A., (2016) Seismic wavefield polarization - Part II: Definition of a parameter system in three-dimensional (3D) space, example case review using LSBB seismic station data. 